\title{
Analysis of bulk and facet failures in AlGaAs-based high-power diode lasers
}

\author{
Jens W. Tomm*a, Martin Hempel ${ }^{\mathrm{a}}$, Fabio La Mattina ${ }^{\mathrm{b}}$, Frank M. Kießling ${ }^{\mathrm{c}}$, Thomas Elsaesser ${ }^{\mathrm{a}}$ \\ ${ }^{\mathrm{a}}$ Max-Born-Institut für Nichtlineare Optik und Kurzzeitspektroskopie, Max-Born-Str. 2A, \\ 12489 Berlin, Germany; ${ }^{b}$ EMPA, Swiss Federal Laboratories for Materials Science and \\ Technology, Überlandstraße 129, 8600 Dübendorf, Switzerland; ${ }^{c}$ Leibniz-Institut für \\ Kristallzüchtung, Max-Born-Straße 2, 12489 Berlin, Germany
}

\begin{abstract}
Mechanisms are addressed limiting the reliability high-power diode lasers. An overview is given on the kinetics of the Catastrophic Optical Damage (COD) process, which is related to highest output powers. It involves fast defect growth fed by re-absorption of laser light. Local temperatures reach the order of the melting temperature of the waveguide of the device. The process starts either at a facet or at any weak point, e.g., at extended defects in the interior of the cavity.
\end{abstract}

Keywords: High-power diode lasers, degradation mechanisms, catastrophic optical damage

\section{INTRODUCTION}

High-power diode lasers are the root-source of photonic energy in most laser systems. Conversion efficiencies exceeding seventy percent make them the most efficient man-made device for converting electric into photonic energy. This importance is motivation for research on how to exploit the entire potential of these lasers. There are principal physical limits: This involves basic properties of the semiconductor materials such as the intraband (momentum) relaxation time and band offsets (barrier heights) [1] setting intrinsic limitations. Extrinsic limits are mainly given by thermal effects resulting among others in deterioration of beam properties reducing the brightness of the devices at highest powers. Moreover, irreversible processes such as degradation become relevant and will be addressed in the following.

Although the history of diode lasers is obviously a story of success, it was always accompanied by device failures. If one reliability problem has been solved, another part of the diode laser became the bottleneck limiting the overall performance on an improved reliability level. The problem of facet degradation has probably been 'solved' several times (and this has been claimed in the literature as well), but the achievement of further increased power levels, again made the facet a problem. The related defect mechanism became called Catastrophic Optical Damage (COD). Meanwhile this effect is not limited to the outcoupling facet, where it has been first discovered in the early days of diode lasers. If the outcoupling facet is well protected, it might appear at rear facets $[2,3]$ or within the bulk [4-6]. Thus COD is rather a potentially limiting mechanism stringently related to high power levels than being only an indication of 'facet problems'.

COD is a sudden degradation process of edge-emitting diode lasers. In involves fast defect growth at local temperatures on the order of the melting temperature of the waveguide of the device. Such temperatures are achieved by localized $r e$ absorption of laser light. The process starts either at a facet or at a weak point, e.g., at an extended defect in the interior of the cavity. It results in a specific defect pattern within the quantum well (QW) plane. COD is not only producing high temperatures, it is also thermally activated. The thermal activation mechanisms are independent from the time scale considered and hold across 15 orders of magnitude on the time scales from nanoseconds to years [7-9].

As mentioned, COD results in specific defect pattern within the QW plane. The appearance of this pattern is absolutely independent on the starting point of COD, facet or bulk. Therefore we do not distinguish between 'Catastrophic Optical Mirror Damage' (COMD) and 'Catastrophic Optical Bulk Damage' (COBD) but use here the common denotation COD. The use of another term than COD seems important in those cases, when, e.g., the bulk damage effect is of different microscopic nature (and produces different types of defect pattern); see e.g. [10].

*tomm@mbi-berlin.de; phone +49-(0)30-63921453; fax 1+49-(0)30-63921459; mbi-berlin.de

Novel In-Plane Semiconductor Lasers XII, edited by Alexey A. Belyanin, Peter M. Smowton,

Proc. of SPIE Vol. 8640, 86401F · (C) 2013 SPIE · CCC code: 0277-786X/13/\$18 · doi: 10.1117/12.2003465 


\section{EXPERIMENTAL}

\subsection{Methodology: Why testing COD in single pulses?}

The main goal of our work is the spatio-temporal resolution of the initiation of the COD and of the subsequent defect propagation. The idea is to measure the effect and to uncover its sequence of events in detail. Before designing the experiment, one should recall the expected time constants being involved into the process. Nakwaski [11-13] dealt with COD modeling based on early work by Henry et al. [14]. He combined three-dimensional analytical modeling with the key assumption of a temperature dependent absorption coefficient. Time constants between 6 and 500 ns were determined. More recent numerical simulations reported by Smith [15] and Miftakhutdinov [16] delivered 10 ns and 10 ns-10 $\mu$ s (depending on the power overload), respectively. We conclude the temporal resolution required to resolve the process to be on the order of $10 \mathrm{~ns}$.

Ideally such investigations should be done in situ during regular operation, e.g. in continuous wave (cw) long-term operation. This approach requires steadily monitoring data. Together with the desired time resolution, this requires $10^{8}$ measurements per second. Although this is feasible for scalar parameters, e.g., emission power or voltage across the laser structure, any more complex information such as the laser nearfield distribution provides excessive amounts of data if continuously monitored. Thus there is need to have a trigger that indicates the COD event in advance. Jacob et al.[17] reported on such work. The authors found in their devices during $\mathrm{cw}$ operation optical and electrical transients occurring prior to COD. Moreover, after initiation of the COD process, they monitored the kinetics of the defect propagation process by high-speed cameras, in particular the motion of 'hot spots' within the QW plane. The temporal resolution was $8.5 \mu \mathrm{s}$. Although this was a great achievement, this time resolution is not sufficient for monitoring the start of the COD and the very early phases of the effect.

Here, we present another approach based on provoking the COD within one single current pulse.

Obviously, this has the disadvantage, that the current required to reach COD exceeds the regular cw operation current. This includes the risk to change the degradation mode that causes the failure. This means if the COD takes place at a substantially increased operation current (or emission power), it could take place in a different way as it would during regular $\mathrm{cw}$ operation. This risk, however, is involved in any type of 'accelerated life testing' that is the base of expected lifetimes given by all device vendors. Therefore, it is very important to verify that COD provoked in single current pulses shows basically the same damage pattern as observed during $\mathrm{cw}$ operation. The advantages of our approach are:

- For pulse lengths on the order of $100 \mathrm{~ns}-100 \mu \mathrm{s}$ the desired temporal resolution of $10 \mathrm{~ns}$ can be achieved, even if complex information such as the emission nearfield is monitored.

- Due to the finite pulse length, the COD process becomes interrupted. This allows preparing earliest phases of COD. As a result, we have available a new quality of degraded devices for microscopic investigations.

- By applying additional pulses, the COD process can be continued (re-ignited). This is a method for time-slicing the process and for monitoring on much longer time scales than $\mu$ s but still maintaining a ns temporal resolution.

- The re-ignition of the COD in a second pulse allows for studying the COD behavior in a situation that is similar to the one in a device being pre-damaged in a defined way.

\subsection{Single-pulse (step) tests: Technical implementations}

Single current pulses are generated by a diode laser driver PicoLas LDP-V50-100 V3. The diode laser is directly attached to the driver in order to guarantee a fast current rise time of $10 \mathrm{~ns}$. The shape of the current transient is monitored by a fast oscilloscope (Agilent Infiniium, $2 \mathrm{GHz}$ ) together with other quantities. This includes:

(a) Emission power transients detected by a fast photodiode Thorlabs Det10A/M (rise time $1 \mathrm{~ns}$ ) together with a timeintegrated thermocamera image (cameras: CEDIP Titanium $560 \mathrm{M}$, Thermosensorik CMT 384) of the front facet $[18,19]$.

(b) Emission power transients were also taken together with a time-integrated thermocamera image of the devices font, rear, and side being additionally imaged by a corner mirror [20], or from the top through a contact window [9].

(c) There were special experiments, where emission power transients have been monitored from front and rear facet at the same time.

(d) The temporal evolution of the laser nearfield was monitored by a Hamamatsu streak camera C1587 (with a single sweep module M1953) [21]. 

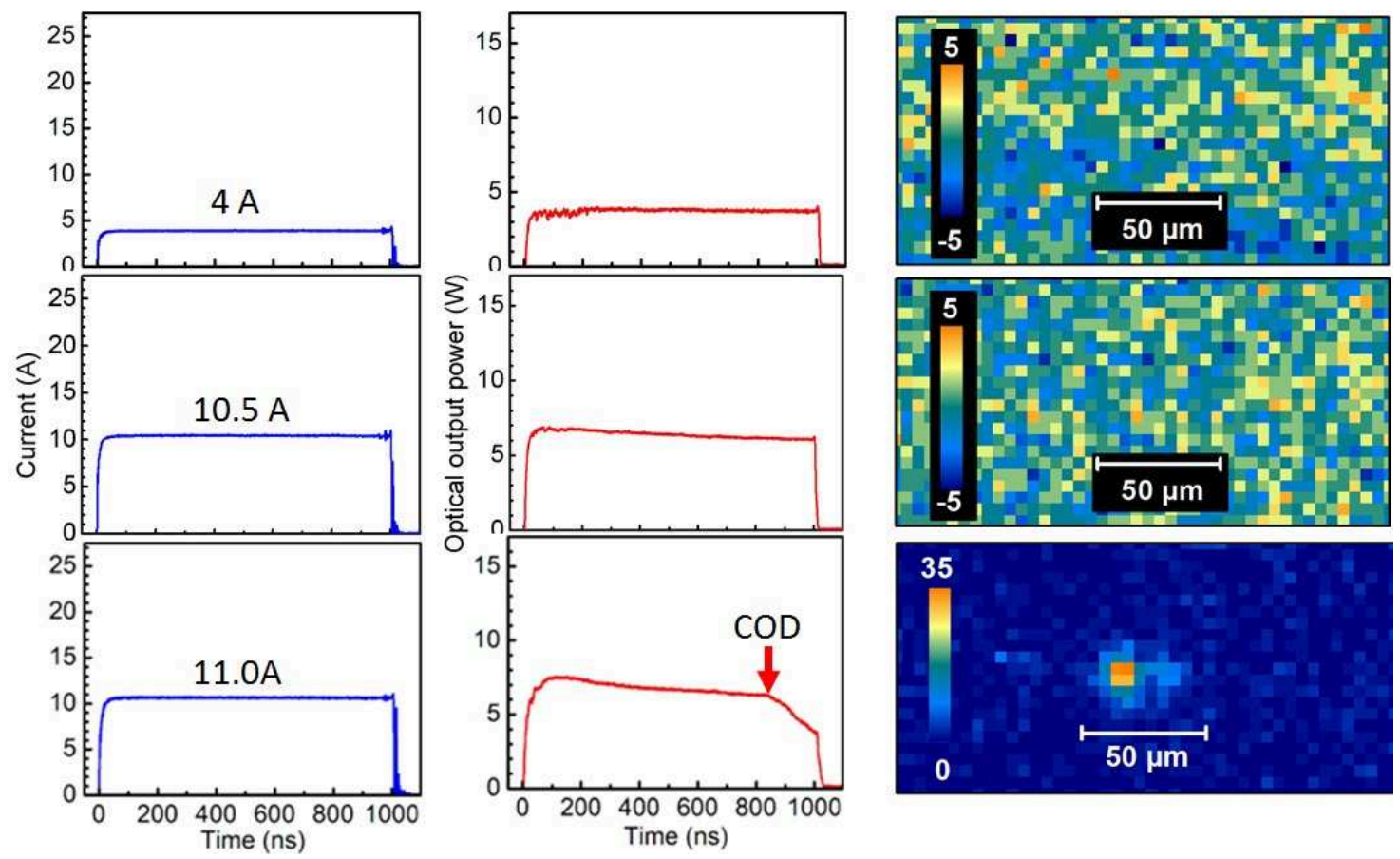

Figure 1. Result of three single-pulse experiments carried out with the same $808 \mathrm{~nm}$ emitting device (from the top to the bottom). While the left column shows the current transients, the middle one gives emission power transients. In the right column, thermocamera images are shown, which have been taken by time-integrating across the full pulse duration (integration time $10 \mu \mathrm{s}$ ). The spatial resolution is better than $8 \mu \mathrm{m}$ and the signal is given in counts.

Figure 1 shows data taken as described above in (a). The full data set includes the current and emission power transients starting at $1.5 \mathrm{~A}$ in 0.5 -A-steps until $\mathrm{COD}$ is observed at $11 \mathrm{~A}$. Comparing the power transients at 4 and $10.5 \mathrm{~A}$, one observes that the power transient develops from a flat shape towards a negative slope. This power loss, which increases continuously from lower to higher currents, represents heat that is additionally stored in the bulk of the waveguide along the entire laser cavity. It leads to an increase of the average waveguide temperature (and thus of the QW temperature) by $\sim 27 \mathrm{~K}$ (from 0 to $1000 \mathrm{~ns}$ ) as determined by measurements of the emission wavelength shift of the device [22]. The waveguide temperature increase has probably been almost the same during the 11-A-pulse; see bottom line in Fig. 1. Nevertheless, a (small) fraction of the energy that is 'missed' in the emission power served to heat a microscopic section near the front facet to the melting point of the material. This is the starting point of the COD. The details will be discussed in section III.

At this point one could ask the question whether the device, which experienced COD at 11 A, see bottom panel in Fig. 1, was still pristine or not. Apparently it underwent a series of 19 pre-pulses, some of them at very high powers, and we can't fully exclude that this induced some gradual aging that could have influenced the COD event observed after $850 \mathrm{~ns}$ within the $20^{\text {th }}$ pulse at $11 \mathrm{~A}$. Nevertheless, this approach seems to be a practical way of COD-testing and we will refer to it from now on as single-pulse step test. One can alternatively perform the same series of experiments with pristine 'nominal identical' devices from the same batch. We reported such work in Ref. [18].

Although the thermocamera provides only time-integrated snapshots, the images give unambiguous evidence that indeed utmost high temperatures are achieved. For the given experimental conditions including the involved spatial and temporal averaging, the noise-equivalent temperature amounts to about $400 \mathrm{~K}$. This well explains, why the $27 \mathrm{~K}$ temperature rise of the entire waveguide, see Fig. 1, second line, is not resolved by the thermocamera, while the CODevent that has taken place in a tiny volume on the order of $1 \mu \mathrm{m}^{3}$ provides a clear signature pointing to a local temperature of about $(1400 \pm 400){ }^{\circ} \mathrm{C}$; see Fig. 1, third line. This type of thermography also allows for the unambiguous identification of COD re-ignition events if another pulse is applied after the initial COD event. 


\section{RESULTS AND DISCUSSION}

\subsection{COD kinetics}

Figure 2 shows an experimental result obtained with a setup as described in subsection 2.2 (d). The device experienced a full single-pulse step test with operation currents from 1.5 to $7.5 \mathrm{~A}$ in $0.5 \mathrm{~A}$ steps (pulse duration of $1 \mu \mathrm{s}$ ). After observing a weak COD signature close to the end of the 7.5-A-pulse, the continuation of the process is monitored in two additional pulses of 7.5 A. The time resolution in this experiment is about $30 \mathrm{~ns}$, and the gain-guided broad-area laser is of the same type as the one for which data are shown in Fig. 1. First we consider the nearfield evolution before COD; see left subfigure. Within the first $200 \mathrm{~ns}$, the nearfield narrows. This is due to the transition from (lateral) gain guiding to index guiding caused by the heating of the area in the emitter stripe [21]. A similar tendency is observed even after COD, see middle and left subfigure.

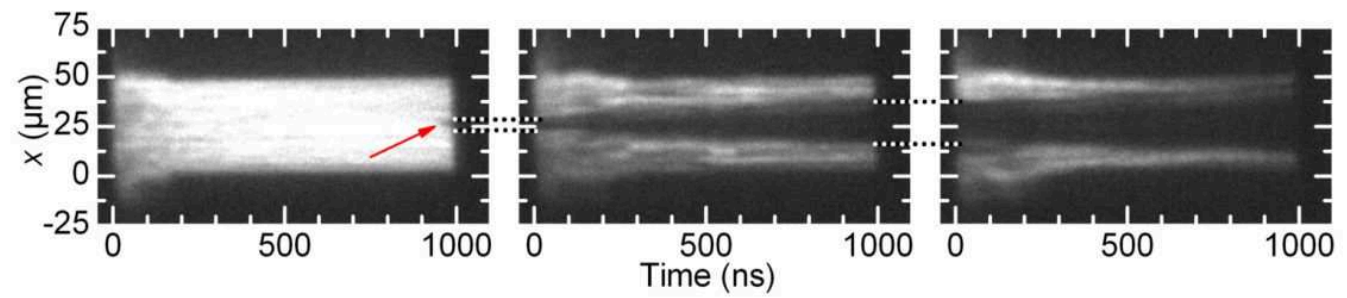

Figure 2. Laser nearfield evolution during COD at a $808 \mathrm{~nm}$ emitting broad-area diode laser. The stripe is $50 \mu \mathrm{m}$ in width. within three successive single 7.5-A-pulses (left to right). The start of the process is indicated in the left emission pattern by an arrow. The dotted lines between the sub-figures indicate that the widths of the gaps are the same at the end of a pulse and the beginning of the successive one.

Close to the end of the first 7.5-A-pulse (left subfigure) the COD-process starts, see arrow. Since the pulse ends shortly after its ignition, the site affected is rather narrow; see dotted lines. The width of this 'dark gap' is the same at the beginning of the following pulse. This supports the concept of time-slicing the process by applying multiple pulses. Furthermore, one can analyze how fast the 'dark gap' is widening. At the beginning of the second pulse this speed is rather small. We interpret this as an accumulation phase of energy that is necessary to re-ignite COD. Although we were not able to check the COD-re-ignition directly by thermography in this particular experiment (see subsection 2.2), we found such evidence in tests with devices from the same batch being operated under similar conditions. Note that after the third pulse the width of the width of the 'dark gap' is larger $(\sim 32.5 \mu \mathrm{m})$ than the width of the signature at the facet $(4.7 \mu \mathrm{m})$ as observed after the test by microscopy.

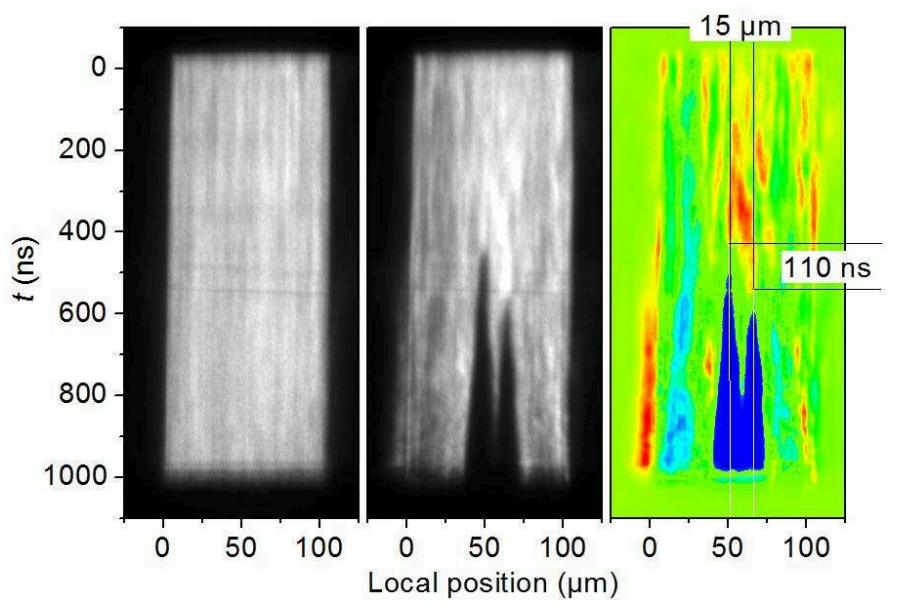

Figure 3. Ignition of COD. Two subsequent single pulses of one $\mu$ s duration from a $780 \mathrm{~nm}$ emitting broad-area diode laser (100 $\mu \mathrm{m}$ stripe width) are shown. Transient emission nearfield is shown in a single pulse of 29.0 A without COD; see left. Transient emission nearfield in a subsequent single pulse of 29.8 A including the COD at two locations; see second from the left. At the right, there is a difference image of the two former ones. Reddish color indicates power increase while blue points to reduction of emission. Visual inspection of the devices revealed two surface defects at the facet being separated by $15 \mu \mathrm{m}$. 
Detailed investigations on this discrepancy revealed that such behavior occurs as long as the optical load at COD is not too high. This is the case in cw but also in single-pulse step tests, where the current steps are small. Here the defect pattern at the front facet remains on the order of a few square microns. The defect serves as the starting point for the creation of a defect network within the plane of the waveguide. This topic has been extensively studied in the literature $[17,23-30]$ and modeled $[4,9,28]$, and will not be further considered here. In case of single-pulse tests with only one high pulse resulting in a high overload at the facet, the damaged front facet area correlates with the power [18, 31]. Thus, the widening of the dark gap in Fig. 2 is interpreted as internal lateral defect growth within the emitter stripe. Lateral defect growth velocities of $\sim 30 \mu \mathrm{m} / \mu \mathrm{s}$ are determined from such pattern [21]. Such high defect propagation velocities represent a key attribute of the COD process.

Figure 3 shows a result obtained in a very similar single-pulse step test. The architecture of the $100 \mu \mathrm{m}$ wide emitter stripe of the broad-area device includes a lateral index step. The device involves built-in index-guiding, and, in contrast to what has been shown in Fig. 2, the width of the emission nearfield is almost constant; see left subfigure. For this particular device, we observe the creation of two COD sites with a temporal delay of $\sim 110 \mathrm{~ns}$. As reported earlier for cw experiments [32], the first COD begins at the position of a strong filament (at $50 \mu \mathrm{m}$ ). Shortly before power reduction takes place at this particular site, the filament moves to the right. We interpret this as a result of the initiating heating (thermal lensing). The filament on the right becomes substantially enhanced ( $\sim 10$ percent) turning this location into a new starting point of a second thermal runaway event (at $65 \mu \mathrm{m}$ ). Thus one degradation event controls a second one via the laser field. The damage pattern eventually observed at the facet show exactly what is expected from the described sequence of events, namely two damaged sites being separated by $\sim 15 \mu \mathrm{m}$. Thus the thermal runaway phase of two COD events is resolved via their emission signature.

\subsection{Analysis of the defect structure}

In order to learn more about COD mechanisms it is desirable to analyze defects in devices, where the energy entered into the defect is small. Devices that experienced COD in one single pulse represent such samples. Figure 4 (left) shows a high-resolution image taken by means of an ORION Helium Ion Microscope (Carl Zeiss). The microscope is based on a He focused ion beam that excites secondary electrons [33]. The device experienced a full single-pulse step test with operation currents from 1.5 to $9 \mathrm{~A}$ in $0.5 \mathrm{~A}$ steps (pulse duration $1.5 \mu \mathrm{s}$ ). COD started $80 \mathrm{~ns}$ before the end of the 9-Apulse. The energy introduced into the defect structure is estimated to $\sim 155 \mathrm{~nJ}$. Taking this energy, the thermal properties of the QW and $\mathrm{Al}_{0.35} \mathrm{Ga}_{0.65} \mathrm{As}$-waveguide, and the apparent volume of the defect (see Fig. 4) one estimates a temporary temperature of some $10^{4} \mathrm{~K}$, i.e., an unrealistic high value. Nevertheless, this number tells us that there was enough energy to reach $1580^{\circ} \mathrm{C}$, the melting point of $\mathrm{Al}_{0.35} \mathrm{Ga}_{0.65} \mathrm{As}$. A striking feature in the microscope image is a 'white line' surrounding severely damaged material, which was found to be caused by AlAs enrichment [33]. Considering the phase diagram of $\mathrm{Ga}_{1-\mathrm{x}} \mathrm{Al}_{\mathrm{x}} \mathrm{As}$ (see scheme in Fig. 4 according to [34]), one understands this observation: After melting of the GaAs-rich waveguide (see dotted line at an AlAs-mole fraction of $x=0.35$ ), segregation leads to the formation of AlAsricher material, which solidifies before material with a lower AlAs-mole fraction (see dotted line at the AlAs-mole fraction of $\mathrm{x}=0.85$ ). This qualitative model explains the formation of such a 'white line', which was observed by other authors as well; see Fig. 5 (left) [35].
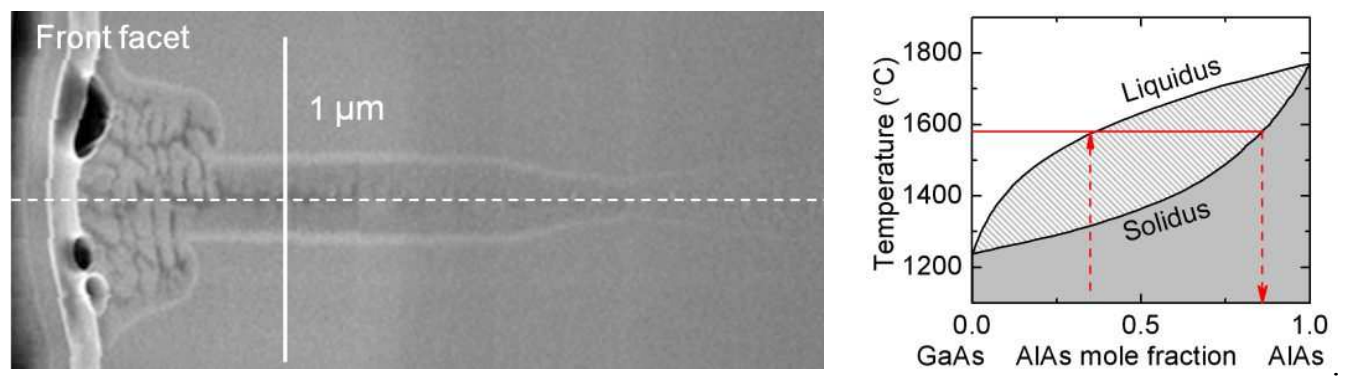

Figure 4. Cross section secondary electron image excited by a Helium Ion Microscope of a device from the batch, which data have been presented in Figs. 1 and 2. The beam current was $0.5 \mathrm{pA}$, the accelerating voltage $30 \mathrm{kV}$. The QW-plane is marked as dashed line, while the vertical bar $(1 \mu \mathrm{m})$ indicates the waveguide thickness. The formerly flat front facet shows a convex shape. 'Black bubbles' are observed between the re-molten semiconductor surface and the partly damaged facet coating. The diagram at the right sketches the phase diagram of the $\mathrm{Ga}_{1-\mathrm{x}} \mathrm{Al}_{\mathrm{x}} \mathrm{As}$ mixed crystal system [34]. 
The white line represents an isothermal line at the border of formerly molten material being created during the recrystallization process after interruption of the COD by the end of the (single) current pulse. Investigations of point defect distributions around primary defects created by COD indeed revealed that the heat is distributed across a substantially larger volume than the one surrounded here by the white line, and still creates point defects there [9]. This also explains the excessive temperature value obtained by the estimate given at the beginning of this discussion. Thus, the area outside the white line is definitely not undamaged. The line is rather the border around the heavily damaged part of the COD pattern.

\subsection{Where does COD start?}

Supposing the white line in Fig. 4 to be indeed an isothermal line, its shape, including the bends, is hardly to be understood assuming one single starting point of the COD-process at the QW-position at the front facet. One could argue that this defect pattern is observed for a special device structure und very special experimental conditions only. Figure 5 (left) shows, however, a very similar pattern obtained from a device that experienced COD during long term cw single spatial mode operation [35]. The device stems from another manufacturer and emits at another wavelength. The common feature of both devices, however, is the $\mathrm{Al}_{1-\mathrm{x}} \mathrm{Ga}_{\mathrm{x}} \mathrm{As}$-waveguide; see discussion above. Since degradation took place in single spatial mode operation, effects are excluded caused by higher order modes, which do not peak at the QW (as the ground mode does). Thus the microstructure of the damage pattern points rather to the waveguide as starting point of COD than to the QW.
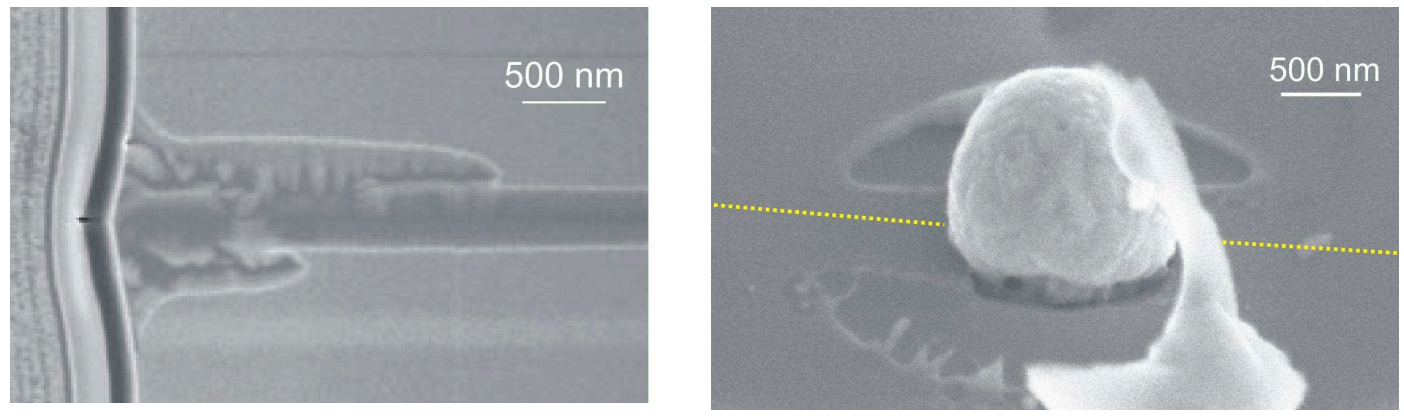

Figure 5. Secondary electron image of a focused ion beam cut through the COD pattern of a 980-nm-emitting single spatial mode device degraded during long-term 'kink-free' cw degradation according to Sin et al. [35] (left). Secondary electron image of the external defect part of a device (tilted by $50^{\circ}$ ); see right image. The position of the QW underneath the front facet coating is indicated by a dotted line. A material extrusion is clearly visible. In addition, the coating is locally affected above and below the QW-plane but not directly on top of the QW. The bright object between the material extrusion and the bottom of the image is a part of the coating that flaked during COD.

A detailed analysis of other damage patterns leads to the same corollary: Figure 5 (right) shows external COD pattern as observed at the front facet of a failed $975-\mathrm{nm}$-emitting device. This particular batch got a not fully optimized front facet coating and here the mirror is seen as the root of degradation. The mushroom-like extrusion in the center is a phenomenon that is more or less pronounced in all COD damage pattern and probably a result of the micro-explosion [36] near the surface, which is involved in COD; at least if it indeed starts at a facet. On top and bottom, however, are the important impacts: On both sides of the QW (dotted line) mirror damage is observed. We see this as the intrinsic root of COD in this batch and again this root is at the waveguide.

Figure 4 shows 'black bubbles' at the waveguide (above and below the QW). We systematically looked for such bubbles and found them in all COD pattern of devices from two batches (from different vendors). We never observed them on top of the QW. We interpret these 'black bubbles' as formed by exhaust of the COD ignition, which again seems to be located at the waveguide.

Recently we studied how external feedback affects the gradual degradation of 808-nm-emitting diode lasers [37]. External feedback is known to be a potential root for diode lasers degradation, which frequently results in COD. This study clearly revealed waveguide and cladding to be affected by the optical overload on a point defect level, but definitely not the QW.

Although none of the four lines of arguments in this subsection alone allows unambiguous assignments, we think that all of them together make it very likely that $G a_{1-x} A l_{x} A$ s-waveguides are likely starting points for COD. 


\subsection{COD inside the cavity: bulk-related COD}

In the preceding section, we located the COD (along growth direction) for those cases, when it starts at a facet. As mentioned, in section 1, COD is an effect related to high power densities, and therefore will start at the device part, which is most susceptible to absorption of laser light. The reason, why so far COD has been mostly studied at facets, was related to two facts: First, except for the case of relatively low emission powers [26, 38], facet-related COD produces externally visible signatures. Second, during the history of diode lasers, facets always represented bottlenecks. Therefore, in particular in the early days, they indeed have been preferential subject of degradation.

Nevertheless, there are reports on bulk-related COD, see e.g. [4-6]. Obviously it is harder to detect, since it starts at an internal site and does not necessarily appear at any surface. Figure 6 shows examples for bulk-related COD.

The first example (left, top) is located about $400 \mu \mathrm{m}$ away from the front facet and was detected by electron beam induced current imaging. This measurement was performed without preparation directly through the top contact of the pup packaged device. Dark areas show regions, where the pn-junction is damaged. The COD-pattern becomes visible as darkened area, since they are located within the depletion region of the pn-junction (see Figs. 4 and 5) of the diode laser structure. The damage suppresses electron-hole separation as a source of the electron beam induced current. The physical origin of this COD event is not detected. Most likely it is an extended defect.

Another example (left, bottom, detected with the same technique) shows three independent extended damage structures, which are created in a single pulse step test ( 42 pulses of $1 \mu$ s duration). The rear facet is directed to the left (at $0 \mu \mathrm{m}$ ). Simultaneously carried out thermocamera measurements provide the sequence of events during creation of this rather complex damage pattern: The process starts at the rear facet and the defect propagation from this facet is finalized after $30 \mu \mathrm{s}$. It ends in a single spot at about $300 \mu \mathrm{m}$. This might be surprising, since one could expect a single spot rather as the starting point. The damaged area in the middle starts after $10 \mu \mathrm{s}$ at the upper edge of the emitter stripe, while the formation of the third damage area begins after $30 \mu$ s, i.e. when the first one is finalized. As in case of the second, also the formation of the third area begins at the upper edge of the emitter stripe. At least the latter two damage areas are examples for bulk-related COD. The starting points of them, the edge of the emitter stripe, is known as area where gradual degradation (point defect formation) can potentially start [39]. Thus for these two areas the root-source of the COD is identified. It is most likely the edge of the emitter stripe metallization. Probably there point defect have been created by processing induced strain in concert with thermo-mechanical stress. Thus a weak point in the design of this particular device architecture is identified by this test.

Figure 6 (right) shows results of a single-pulse step test carried out with a single spatial mode device. Here thermocamera images taken from the side of the $3.9 \mathrm{~mm}$ long device are shown. While the upper subfigure shows a thermal COD signature about $1.2 \mathrm{~mm}$ behind the front facet, the following current pulse shows COD-related defect propagation into both directions. The huge spatial extension of the signature is due to the rather long current pulses.

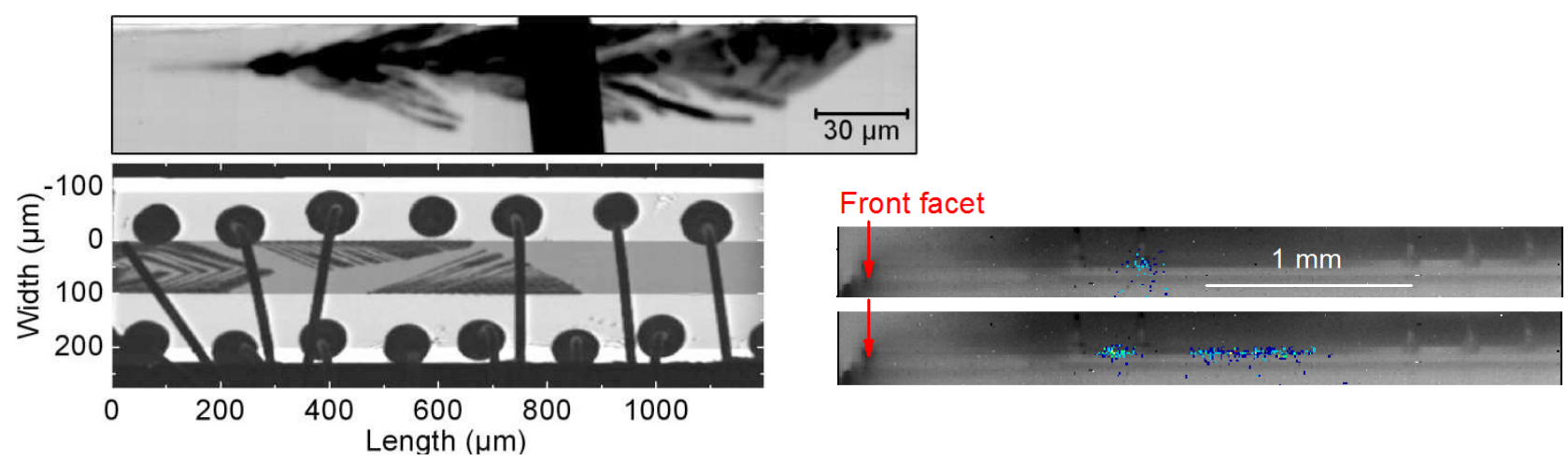

Figure 6. Electron beam induced current images of damage pattern in emitter stripes of two $975 \mathrm{~nm}$ emitting high power diode lasers according to [4]; see left subfigures. The about $25 \mu \mathrm{m}$ wide black gap in the upper subfigure is the shadow from a bond wire. In the lower figure, bond wires crossing the emitter stripe are well visible. The right subfigures represent composite thermal images taken from the side of a single spatial mode diode laser during two single high-power current pulses of 8.2A (right top) and 8.7A (right bottom) of $9 \mu$ s duration according to [40]. Each image consists of two parts: There is an emissivity contrast image, which reveals the device geometry, see, e.g., bond wires (front facet is marked by an arrow). This image is taken without device operation (black/white contrast, integration time $1 \mathrm{~ms}$ ), while the color-coded overlayed image reveals the thermal radiation (integration time $10 \mu$ s triggered in a way to cover the entire pulse duration). 
Internal defect generation and growth has also been observed in quantum dot lasers [10]. Nevertheless, we think that the degradation effects observed there should not be called COD because there is no evidence for fast defect propagation fed by re-absorbed laser light.

\section{SUMMARY AND CONCLUSIONS}

We discuss the COD effect as a specific sudden degradation mechanism related to high power densities and leading to damage pattern, which propagation is fed by laser light. The starting point can be either at one of the facets or in the bulk. Although manufacturing technology continuously improved and lead to excellent protected surfaces and lower defect densities in the bulk, increasing power levels compensate for these achievements, and again promote this type of degradation. Because of its intrinsic link to high power densities, COD will always remain one potential limitation on the way toward increasing emission powers from diode lasers.

Case studies are discussed, where COD takes place at facets or in the bulk. We find strong indication that if COD starts at the outcoupling facet of AlGaAs-based lasers its origin is rather at the $\mathrm{Ga}_{1-\mathrm{x}} \mathrm{Al}_{\mathrm{x}} \mathrm{As}$-waveguide than at the QW. We show that the COD effect takes place at local temperatures of $\sim 1500^{\circ} \mathrm{C}$.

We propose single-pulse step tests for COD analysis and demonstrate its potential for the creation of early stages of COD and accelerated life testing. In order to justify this approach, we show that damage pattern created in a single-pulse step test and generated during cw operation look similar.

- This holds for the nm scale; compare Figs. 4 (aged in a single pulse) and 5 (cw aging test).

- Considering the $\mu \mathrm{m}$ scale one can compare the defect networks within the plane of the waveguide. These defect networks are not topic of this report. However the appearance of those, which are created in standard tests [17, 23$30]$ and in single-pulse step tests $[4,9]$, are absolutely similar.

Therefore we consider it very likely that COD in broad-area lasers takes place in the same way during single-pulse step tests and cw operation.

What are the limitations of single-pulse tests?

- Considering broad-area lasers, we haven't seen any limitations in step tests. Only in case that single pulses of extremely high amplitude have been applied (i.e. no stepwise approach towards the COD threshold) [18], we find modifications of the dominating degradation mechanism. This, however, offers also the option to provoke and investigate COD in devices, which typically do not fail by COD during regular cw operation. Thus this limit can also be turned into an additional option.

- We conducted COD step tests in single spatial mode devices, which typically fail by facet-related COD during longterm regular cw operation in fundamental mode [40]. At utmost high overload in single pulses, they rather went into multiple-spatial-mode operation than failed by COD. Thus the single-pulse step test was not applicable here for testing facet-related COD.

- Quantum-dot devices have been analyzed as well [10]. During single pulse excitation dark, non-radiative spots have been observed in the bulk, which enlarge as pulse length increases, but do not show the type of fast defect propagation as discussed before.

Knowledge about COD kinetics is important because it is a key mechanism, which is stringently related to high emission powers. This study should eventually help finding strategies that help delaying COD towards even higher emission powers.

\section{ACKNOWLEGEMENT}

The authors would like to thank Juan Jimenez and Michel Krakowski for helpful discussions and Ivan Shorubalko and Michael Stiefel for device preparation. The authors thank SNSF R'equip for the possibility to use the He Ion Microscope at EMPA. Funding by the European Commission within the Integrated Project WWW.BRIGHTER.EU, contract No. 035266, is acknowledged. 


\section{REFERENCES}

[1] X. Z. Wang, P. Crump, H. Wenzel, A. Liero, T. Hoffmann, A. Pietrzak, C. M. Schultz, A. Klehr, A. Ginolas, S. Einfeldt, F. Bugge, G. Erbert, and G. Trankle, "Root-Cause Analysis of Peak Power Saturation in PulsePumped $1100 \mathrm{~nm}$ Broad Area Single Emitter Diode Lasers," IEEE Journal of Quantum Electronics, 46(5), 658665 (2010).

[2] M. Hempel, J. W. Tomm, M. Ziegler, T. Elsaesser, N. Michel, and M. Krakowski, "Catastrophic optical damage at front and rear facets of diode lasers," Applied Physics Letters, 97(23), 231101 (2010).

[3] U. Menzel, "Self-consistent calculation of facet heating in asymmetrically coated edge emitting diode lasers," Semiconductor Science and Technology, 13(3), 265-276 (1998).

[4] M. Hempel, J. W. Tomm, V. Hortelano, N. Michel, J. Jimenez, M. Krakowski, and T. Elsaesser, "Timeresolved reconstruction of defect creation sequences in diode lasers," Laser \& Photonics Reviews, 6(6), L15L19 (2012).

[5] Y. Sin, N. Presser, B. Foran, N. Ives, and S. C. Moss, "Catastrophic Facet and Bulk Degradation in High Power Multi-Mode InGaAs Strained Quantum Well Single Emitters," SPIE Proc., 7198, 719818-1 - 719818-12 (2009).

[6] A. K. Chin, R. K. Bertaska, H. Eppich, M. A. Jaspan, and J. H. Jacob, "Bulk catastrophic optical-damage of 980 nm InxGa1-xAs/GaAs high-power, broad-area laser-diodes due to a void on Au80S20n solder," Mater. Res. Soc. Symp. Proc., 1195, B02-03 (2010).

[7] A. Moser, and E. E. Latta, "Arrhenius parameters for the rate-process leading to catastrophic damage of AlGaAs laser facets," Journal of Applied Physics, 71(10), 4848-4853 (1992).

[8] A. Moser, E. E. Latta, and D. J. Webb, "Thermodynamics approach to catastrophic optical mirror damage of AlGaAs single quantum well lasers," Applied Physics Letters, 55(12), 1152-1154 (1989).

[9] M. Hempel, F. La Mattina, J. W. Tomm, U. Zeimer, R. Broennimann, and T. Elsaesser, "Defect evolution during catastrophic optical damage of diode lasers," Semiconductor Science and Technology, 26(7), 075020 (2011).

[10] S. N. Elliott, M. Hempel, U. Zeimer, P. M. Smowton, and J. W. Tomm, "Catastrophic optical bulk damage in InP 7xx emitting quantum dot diode lasers," Semiconductor Science and Technology, 27(10), 102001 (2012).

[11] W. Nakwaski, "Thermal analysis of the catastrophic optical damage in laser diodes," Journal of Applied Physics, 57(7), 2424-2430 (1985).

[12] W. Nakwaski, "Three-dimensional time-dependent thermal model of catastrophic mirror damage in stripegeometry double-heterostructure GaAs/(AlGa)As diode lasers " Optical and Quantum Electronics, 21(4), 331334 (1989).

[13] W. Nakwaski, "Thermal model of the catastrophic degradation of high-power stripe-geometry GaAs/(AlGa)As double-heterostructure diode lasers," Journal of Applied Physics, 67(4), 1659-1668 (1990).

[14] C. H. Henry, P. M. Petroff, R. A. Logan, and F. R. Merritt, "Catastrophic Damage of $\mathrm{Al}_{\mathrm{x}} \mathrm{Ga}_{1-\mathrm{x}} \mathrm{As}$ DoubleHeterostructure Laser Material," Journal of Applied Physics, 50(5), 3721-3732 (1979).

[15] W. R. Smith, "Mathematical modeling of thermal runaway in semiconductor laser operation," Journal of Applied Physics, 87(12), 8276-8285 (2000).

[16]D. R. Miftakhutdinov, A. P. Bogatov, and A. E. Drakin, "Catastrophic optical degradation of the output facet of high-power single-transverse-mode diode lasers. 2. Calculation of the spatial temperature distribution and threshold of the catastrophic optical degradation," Quantum Electronics, 40(7), 589-595 (2010).

[17] J. H. Jacob, R. Petr, M. A. Jaspan, S. D. Swartz, M. T. Knapczyk, and A. M. Flusberg, "Fault protection of broad-area laser diodes," SPIE Proceedings, 7198, 719815 (2009).

[18] M. Ziegler, J. W. Tomm, D. Reeber, T. Elsaesser, U. Zeimer, H. E. Larsen, P. M. Petersen, and P. E. Andersen, "Catastrophic optical mirror damage in diode lasers monitored during single-pulse operation," Applied Physics Letters, 94(19), 191101 (2009).

[19] M. Ziegler, M. Hempel, H. E. Larsen, J. W. Tomm, P. E. Andersen, S. Clausen, S. N. Elliott, and T. Elsaesser, "Physical limits of semiconductor laser operation: A time-resolved analysis of catastrophic optical damage," Applied Physics Letters, 97(2), 021110 (2010).

[20] M. Hempel, M. Ziegler, J. W. Tomm, T. Elsaesser, N. Michel, and M. Krakowski, "Time-resolved analysis of catastrophic optical damage in $975 \mathrm{~nm}$ emitting diode lasers," Applied Physics Letters, 96(25), 251105 (2010).

[21] M. Hempel, J. W. Tomm, M. Baeumler, H. Konstanzer, J. Mukherjee, and T. Elsaesser, "Near-field dynamics of broad area diode laser at very high pump levels," AIP Advances, 1(4), 042148 1-6 (2011). 
[22] M. Hempel, J. W. Tomm, M. Baeumler, H. Konstanzer, J. Mukherjee, and T. Elsaesser, "Near-field characteristics of broad area diode lasers during catastrophic optical damage failure," SPIE Proc., 8432, 84320 O-843200 (2012).

[23] C. W. Snyder, J. W. Lee, R. Hull, and R. A. Logan, "Catastrophic degradation lines at the facet of InGaAsP/InP lasers investigated by transmission electron microscopy," Applied Physics Letters, 67(4), 488-490 (1995).

[24] I. Rechenberg, A. Hopner, J. Maege, A. Klein, G. Beister, and M. Weyers, "Heating and damage of InGaAs/GaAs/AlGaAs laser facets," Institute of Physics Conference Series, Microscopy of Semiconducting Materials 1995, 146, 587-590 (1995).

[25] R. E. Mallard, and R. Clayton, "EBIC and TEM analysis of catastrophic optical damage in high power GaAlAs/GaInAs lasers," SPIE Proceedings, 3004, 145-150 (1997).

[26] M. BouSanayeh, A. Jaeger, W. Schmid, S. Tautz, P. Brick, K. Streubel, and G. Bacher, "Investigation of dark line defects induced by catastrophic optical damage in broad-area AlGaInP laser diodes," Applied Physics Letters, 89(10), (2006).

[27] T. S. Yeoh, J. A. Chaney, M. S. Leung, N. A. Ives, Z. D. Feinberg, J. G. Ho, and J. U. Wen, “Threedimensional failure analysis of high power semiconductor laser diodes operated in vacuum," Journal of Applied Physics, 102(12), (2007).

[28] A. K. Chin, R. K. Bertaska, M. A. Jaspan, A. M. Flusberg, S. D. Swartz, M. T. Knapczyk, R. Petr, I. Smilanski, and J. H. Jacob, "A comprehensive model of catastrophic optical-damage in broad-area laser diodes," SPIE Proceedings, 7198, 71981A (2009).

[29] M. Baeumler, J. L. Weyher, S. Muller, W. Jantz, R. Stibal, G. Herrmann, J. Luft, K. Sporrer, and W. Spath, "Investigation of degraded laser diodes by chemical preparation and luminescence microscopy," Defect Recognition and Image Processing in Semiconductors DRIP VII 1997, 160, 467-470 (1998).

[30] C. Frigeri, M. Baeumler, A. Migliori, S. Muller, J. L. Weyher, and W. Jantz, "Optical and structural analysis of degraded high power InGaAlAs/AlGaAs lasers," Materials Science and Engineering B-Solid State Materials for Advanced Technology, 66(1-3), 209-214 (1999).

[31] S. N. Elliott, P. M. Smowton, M. Ziegler, J. W. Tomm, and U. Zeimer, "Time resolved studies of catastrophic optical mirror damage in red-emitting laser diodes," Journal of Applied Physics, 107(12), 123116 (2010).

[32] M. BouSanayeh, P. Brick, W. Schmid, B. Mayer, M. Muller, M. Reufer, K. Streubel, S. Schwirzke-Schaaf, J. W. Tomm, A. Danilewsky, and G. Bacher, "Defect investigation and temperature analysis of high-power AlGaInP laser diodes during catastrophic optical damage," Journal of Materials Science-Materials in Electronics, 19, S155-S159 (2008).

[33] M. Hempel, J. W. Tomm, F. L. Mattina, I. Ratschinski, M. Schade, I. Shorubalko, M. Stiefel, H. S. Leipner, F. M. Kießling, and T. Elsaesser, "Microscopic Origins of Catastrophic Optical Damage in Diode Lasers," IEEE Journal of Selected Topics in Quantum Electronics, in print (2013).

[34] L. M. Foster, J. E. Scardefield, and J. F. Woods, "Thermodynamic Analysis of III-V Alloy Semiconductor Phase Diagrams: III . The Solidus Boundary in the Pseudobinary System,” Journal of the Electrochemical Society, 119(6), 765-766 (1972).

[35] Y. Sin, N. Presser, B. Foran, M. Mason, and S. C. Moss, "Investigation of Catastrophic Optical Mirror Damage in High Power Single-Mode InGaAs-AlGaAs Strained Quantum Well Lasers with Focused Ion Beam and HRTEM Techniques," 2007 Conference on Lasers \& Electro-Optics/Quantum Electronics and Laser Science Conference (CLEO/QELS 2007), Vols 1-5, 1423-1424 (2007).

[36] P. G. Eliseev, “Degradation of injection lasers,” Journal of Luminescence, 7, 338-356 (1973).

[37] M. Hempel, M. Chi, P. M. Petersen, U. Zeimer, and J. W. Tomm, "How does external feedback cause AlGaAsbased diode lasers to degrade?," Applied Physics Letters, in print (2013).

[38] M. BouSanayeh, P. Brick, W. Schmid, B. Mayer, M. Muller, M. Reufer, K. Streubel, J. W. Tomm, and G. Bacher, "Temperature-power dependence of catastrophic optical damage in AlGaInP laser diodes," Applied Physics Letters, 91(4), 041115 (2007).

[39] M. Ziegler, T. Q. Tien, S. Schwirzke-Schaaf, J. W. Tomm, B. Sumpf, G. Erbert, M. Oudart, and J. Nagle, "Gradual degradation of red-emitting high-power diode laser bars," Applied Physics Letters, 90(17), 171113 (2007).

[40] M. Hempel, J. W. Tomm, T. Elsaesser, and M. Bettiati, "High single-spatial-mode pulsed power from $980 \mathrm{~nm}$ emitting diode lasers,” Applied Physics Letters, 101(19), 191105 (2012). 